\title{
Machine Learning-Based Handovers for Sub-6 GHz and mmWave Integrated Vehicular Networks
}

\author{
Li Yan ${ }^{\circledR}$, Haichuan Ding ${ }^{\circledR}$, Lan Zhang ${ }^{\circledR}$, Jianqing Liu ${ }^{\circledR}$, Xuming Fang ${ }^{\circledR}$, Senior Member, IEEE, \\ Yuguang Fang ${ }^{(}$, Fellow, IEEE, Ming Xiao ${ }^{(}$, Senior Member, IEEE, \\ and Xiaoxia Huang, Member, IEEE
}

\begin{abstract}
The integration of sub-6 $\mathrm{GHz}$ and millimeter wave (mmWave) bands has a great potential to enable both reliable coverage and high data rate in future vehicular networks. Nevertheless, during mmWave vehicle-to-infrastructure (V2I) handovers, the coverage blindness of directional beams makes it a significant challenge to discover target $\mathrm{mmWave}$ remote radio units (mmW-RRUs) whose active beams may radiate somewhere that the handover vehicles are not in. Besides, fast and soft handovers are also urgently needed in vehicular networks. Based on these observations, to solve the target discovery problem, we utilize channel state information (CSI) of sub-6 GHz bands and Kernel-based machine learning (ML) algorithms to predict vehicles' positions and then use them to pre-activate target mmW-RRUs. Considering that the regular movement of vehicles on almost linearly paved roads with finite corner turns will generate some regularity in handovers, to accelerate handovers, we propose to use historical handover data and $K$-nearest neighbor (KNN) ML algorithms to predict handover decisions without involving time-consuming target selection and beam training processes. To achieve soft handovers, we propose to
\end{abstract}

Manuscript received September 23, 2018; revised January 31, 2019 , April 6, 2019 and June 13, 2019; accepted July 16, 2019. Date of publication July 29, 2019; date of current version October 9, 2019. The work of L. Yan and $X$. Fang was partially supported by the NSFC Guangdong Joint Foundation under Grant U1501255, and the NSFC High-Speed Rail Joint Foundation under Grant U1834210 The work of H. Ding, L. Zhang, and Y. Fang was partially supported by the US National Science Foundation under Grant CNS-1343356. The work of M. Xiao was partially supported by the Swedish Strategic Foundation (SSF) through the project (High-reliable Low-latency Industrial Wireless Communications), the EU Marie Sklodowska-Curie Actions through the project (High-reliability Low-latency Communications with network coding), and ERA-NET (SMART-MLA). The work of X. Huang was partially supported by the NSFC Guangdong Joint Program under Grant U1501255 and the Guangdong Special Support for Young Innovation Talents under Grant 2016TQ03X279. The associate editor coordinating the review of this paper and approving it for publication was J. Liu. (Corresponding author: Li Yan.)

L. Yan and X. Fang are with the Key Laboratory of Information Coding and Transmission, Southwest Jiaotong University, Chengdu 610031, China (e-mail: liyan12047001@my.swjtu.edu.cn; xmfang@swjtu.edu.cn).

H. Ding is with the Department of Electrical Engineering and Computer Science, University of Michigan, Ann Arbor, MI 48109 USA (e-mail: dhcbit@gmail.com).

L. Zhang and Y. Fang are with the Department of Electrical and Computer Engineering, University of Florida, Gainesville, FL 32611 USA (e-mail: lanzhang@ufl.edu; fang@ece.ufl.edu).

J. Liu is with the Department of Electrical and Computer Engineering, The University of Alabama in Huntsville, Huntsville, AL 35899 USA (e-mail: jianqing.liu@uah.edu).

M. Xiao is with the Department of Communication Theory, Royal Institute of Technology, 10044 Stockholm, Sweden (e-mail: mingx@kth.se).

$\mathrm{X}$. Huang is with the School of Electronics and Communication Engineering, Sun Yat-sen University, Guangzhou 510006, China (e-mail: huangxiaoxia@mail.sysu.edu.cn).

Color versions of one or more of the figures in this article are available online at http://ieeexplore.ieee.org.

Digital Object Identifier 10.1109/TWC.2019.2930193 employ vehicle-to-vehicle (V2V) connections to forward data for V2I links. The theoretical and simulation results are provided to validate the feasibility of the proposed schemes.

Index Terms-Control/user-plane decoupling, vehicular networks, handovers, target discovery, machine learning, V2V communications.

\section{INTRODUCTION}

$\mathbf{I}$ N FUTURE Intelligent Transportation Systems (ITSs), vehicles will be equipped with more advanced sensors to monitor surrounding driving environments, driving behaviors and vehicle status, and share the collected data through vehicular networks to facilitate traffic management and improve driving experience [1], [2]. On the other hand, passengers in vehicles also desire wireless connections to enjoy high-quality mobile services just as at home. Obviously, the evolution of ITSs has raised ultra-high capacity requirements to future vehicular networks. Recently, to meet the ever increasing data-rate demands, many efforts from both academia and industries have been made to tackle the difficulties of applying extremely broadband mmWave bands in $5 \mathrm{G}$ communications, including vehicular scenarios [3]. To compensate the severe path loss in mmWave bands, energy-concentrated directional beamforming has been widely applied as an indispensable technology in mmWave communications to extend the signal propagation distance [4]. Nevertheless, the coverage scope of directional beams is significantly limited, which will definitely result in the coverage blindness problem. Depending only on mmWave communications cannot meet the strict transmission reliability requirement of critical vehicular services, such as driving safety related services. Based on this observation, in this paper, we employ the control/user-plane (C/U-plane) decoupled network architecture which integrates high-quality lower frequency bands (such as conventional cellular sub$6 \mathrm{GHz}$ bands) with broadband higher frequency bands (such as mmWave bands) to guarantee the coverage performance while expanding bandwidth [5]. Under this network architecture, omni-directional connections on lower frequency bands can not only assist beam alignments on mmWave bands, but also take over transmissions when mmWave connections are interrupted, which to a large extent enhances the robustness of the whole vehicular network.

Nevertheless, even under the sub-6 GHz and mmWave integrated network, the inherent coverage blindness of mmWave directional beams still challenges the target discovery processes during handovers. Typically, there are two kinds 
of wireless connections in vehicular networks, i.e., vehicleto-vehicle (V2V) and vehicle-to-infrastructure (V2I) links [6]. Owing to the short wavelength of mmWave bands, it is reasonable to assume that massive antenna arrays are accommodated in roadside mmWave remote radio units (mmW-RRUs) to simultaneously serve multiple vehicles. During V2I transmissions, the active beams of an mmW-RRU are radiated towards in-service vehicles while other beams are silent. As a result, the effective coverage of an mmW-RRU will be narrow spotlike areas around its in-service vehicles. In such a situation, once a vehicle requires to hand over to this mmW-RRU, it can detect this mmW-RRU only when it happens to be in this effective area. Otherwise, the handover vehicle will miss this candidate mmW-RRU which may be the best choice. In the worst case, the handover vehicle even cannot detect any candidate mmW-RRUs and has to reconnect to the network. To overcome the target discovery problem during handovers in mmWave networks, neighboring candidate mmW-RRUs of handover vehicles should be pro-actively prepared, where the position information of vehicles becomes a key factor. There are several approaches to obtain accurate and timely position information of vehicles. In this paper, we use the channel state information (CSI) over sub-6 GHz bands as the input feature data of the Kernel-based ML algorithm to predict vehicles' positions. Since CSI has to be collected for control purposes anyhow in any wireless communication systems, compared with conventional trajectory estimation methods where some movement information needs to be fed back to the network side [7], [8], the positioning method used in this paper will not cause any extra feedback overheads. Based on the position information, once a vehicle requests V2I handovers, lower frequency RRUs (LF-RRUs) operating at sub-6 GHz bands will pre-activate the candidate $\mathrm{mmW}$-RRUs near this vehicle, thereby overcoming the target discovery problem caused by the coverage blindness of mmWave directional beams.

Under high-mobility environments where users rapidly pass through handover overlapping areas, there is no sufficient time left to accomplish handovers, which will get even worse when considering the complicated beam training processes during handovers in mmWave communications. Effective approaches to achieve fast handovers in this scenario are of urgent need. Intuitively, in vehicular scenarios, on approximately linearlypaved roads with a finite number of corner turns, the regular mobility patterns of vehicles will bring in certain regularity in handovers [9]. Based on this observation, to realize fast V2I handovers, we first record the status information of vehicles when requesting handovers and the final handover decisions during V2I handovers and then use the $K$-nearest neighbor ML algorithm to exploit their internal relationship [10]. Afterwards, by utilizing the status information of vehicles at the time of requesting handovers, LF-RRUs can directly make handover decisions without involving the complicated and time-consuming target selection and beam training procedures, thereby accelerating V2I handovers.

The widely adopted handover in current wireless communication networks is the break-before-make hard handover [11], which inevitably causes temporary transmission interruptions during handovers. To realize interruption-free V2I handovers, we propose to use massive available $\mathrm{V} 2 \mathrm{~V}$ links to forward data for V2I links during handovers. On future advanced vehicles, in addition to the roof, mmWave transceivers can also be deployed on four sides of the vehicle body to gain more opportunities of establishing $\mathrm{V} 2 \mathrm{~V}$ links to release the traffic burden on V2I links. Compared with V2I links, V2V links built between co-directional vehicles are more stable because of low relative velocities. Since in this paper V2V links are established to forward data for V2I links during handovers, we can always consciously choose co-directional vehicles to guarantee the V2V link stability.

In this paper, considering the regularities behind handovers, ML algorithms, which have a great ability to unravel the internal relationship between the inputs and outputs of a system, are utilized to solve the aforementioned handover problems in mmWave vehicular networks. It should be noted that this paper focuses on how to use ML algorithms to assist handovers in mmWave vehicular networks, instead of performance improvements for ML algorithms themselves. Therefore, we only adopt two typical algorithms, i.e., the Kernel-based ML algorithm for positioning and the KNN ML algorithm for handover decision predictions. Nevertheless, more powerful algorithms can also be used to improve the performance of the whole scheme.

The main contributions of this paper can be listed as follows.

1) To expand the system bandwidth as well as guarantee the coverage performance, we apply the C/U-plane decoupled network architecture in vehicular networks to enable the integration of both broadband mmWave bands and omnidirectional sub- $6 \mathrm{GHz}$ bands.

2) To solve the target mmW-RRU discovery problem due to the coverage blindness of directional beams, we propose to utilize the already collected CSI on sub- $6 \mathrm{GHz}$ bands and the Kernel-based ML algorithm to predict vehicles' positions, which are later used by LF-RRUs to pre-activate the candidate mmW-RRUs near handover vehicles.

3) To accelerate handovers, we utilize historical handover data and the KNN ML algorithm to determine the internal relationship between the status information of vehicles when requesting handovers and the final handover decisions. Based on the derived relationship and the status information of vehicles at the time of requesting handovers, LF-RRUs can directly give handover decisions without involving complicated target selection and beam training processes.

4) To achieve soft handovers, we develop a V2V-assisted handover mechanism, in which a purposefully established $\mathrm{V} 2 \mathrm{~V}$ link with relatively high connection stability is employed to forward data for vehicles that are executing V2I handovers to avoid transmission interruptions.

The rest of this paper is organized as follows. In Section II, some related works are investigated. In Section III, the handover problems under the sub- $6 \mathrm{GHz}$ and mmWave integrated network architecture are presented. In Section IV, the machine learning based handover schemes are discussed in details. In Section V, mathematical models are formulated to illustrate the proposed schemes. In Section VI, simulation results are presented to validate the feasibility of the proposed schemes. Finally, Section VII concludes this paper. 


\section{RELATED WORK}

To meet the ever-increasing capacity requirements, as well as to guarantee the mobility performance, as stated in [12], future vehicular networks need to be heterogeneous, which combine BSs with different capabilities into one network. In [13], Ding et al. proposed a vehicular cognitive capability harvesting network (V-CCHV) with separate control, data and access planes to facilitate the network management. They also pointed out that in future vehicular networks, roadside BSs will be equipped with abundant computing resources and storage space to support the communication requirements. In [14], $\mathrm{Wu}$ et al. integrated the software defined network and C/Uplane decoupled architecture into future vehicular networks to improve the mobility management.

To solve the target discovery problem which will be explained in details in Section III.B, the position information of vehicles becomes a key factor. Nevertheless, in conventional trajectory estimation algorithms, such as the Kalman filter method mentioned in [7], [8], some currently observed state condition of the target object, such as the position or velocity information, is required to predict the next state condition. Nevertheless, the transmission of these information will consume extra wireless resources. Additionally, wireless networks can also track the trajectory of vehicles directly by using ML algorithms to exploit the internal relationship between the constantly-collected wireless signal measurements and vehicles' positions. In [15], as an effective method, the low-complex Kernel-based ML algorithm was utilized to take the received signal strength indicator (RSSI) as input to localize and track the mobile targets in wireless sensor networks. Nevertheless, it was pointed out in [16] that RSSIbased positioning systems suffer from dramatic performance degradation in complex situations due to multi-path fading and temporal dynamics. In [17], the field test results revealed that CSI values of multiple sub-carriers exhibit great stability over time at the same position but apparent variability at different positions. Based on this observation, Wang et al. in [17] proposed to use CSI as the input of ML algorithms to locate users.

Traditionally, the handover decision metrics span a wide range of network parameters, such as the RSSI, word error indicator (WEI), quality indicator (QI), among which the metric of RSSI is the basic concern [18]. Theoretically, we normally use RSSI as the only decision metric. Besides, to avoid the ping-pong effect that an user recurrently hands over and back between two adjacent BSs due to the fluctuation of wireless signals, the handover is triggered only when the signal strength received from the target $\mathrm{BS}$ is $\Gamma \mathrm{dB}$ higher than that from the source BS for a predefined duration, namely time to trigger (TTT) [19], where $\Gamma$ is the handover threshold. Nevertheless, under high-mobility scenarios, the conventional time-consuming handover decision-making method will aggravate the insufficient handover time problem, which is considered and solved in [20] by using different TTT levels to adapt to different velocities. In [21], user movement patterns learnt through machine learning techniques are used as a key reference to adjust the handover parameters. In their scheme, both the signal quality and traffic loads of the target base stations are taken into account when determining the optimal handover parameters. Currently, most works focus on the handover process of sub- $6 \mathrm{GHz}$ wireless systems. Researches on the handover of directional mmWave communications are still in the infancy phase. In [22], a machine learning based handover decision scheme is proposed to improve the vertical handovers between the micro and mmWave bands. Nevertheless, the handovers between mmWave base stations are not discussed. In [23], the inter-beam handover within a serving mmWave base station is studied without considering the inter-site handovers. In this paper, our study focus is on the inter-site handovers of mmWave directional communications. To mitigate the problem of insufficient handover time under high-mobility vehicular scenarios, instead of going through the traditional time-consuming handover metric judgment processes and beam alignments, we use machine learning algorithms to derive the handover regularity from the historical handover data and then directly give out handover decisions, thereby achieving fast handovers.

\section{Network Architecture AND V2I HANDOVER PROBLEMS}

In this section, we first present the C/U-plane decoupled network architecture that integrates sub- $6 \mathrm{GHz}$ bands with broadband mmWave bands. Under this architecture, the V2I handover problems are elaborated and analyzed.

\section{A. Sub-6 GHz and mmWave Integrated Networks}

Driving safety related services have raised strict transmission reliability requirements to future vehicular networks, which should be carefully considered especially when introducing unreliable but broadband mmWave communications to enhance the system capacity. Therefore, in this paper, we adopt the C/U-plane decoupled network architecture which combines both reliable sub- $6 \mathrm{GHz}$ bands to guarantee the coverage performance and the unreliable but broadband mmWave bands to expand the system bandwidth. To facilitate the understanding, in the following we take LTE protocols as a baseline to describe this network. To improve the flexibility in network deployments and the efficiency of baseband resource usage, as shown in Fig. 1, network nodes are connected in the form of cloud radio access networks (C-RANs). The two types of RRUs, i.e., LF-RRUs and mmW-RRUs respectively operating at sub- $6 \mathrm{GHz}$ bands and mmWave bands, are deployed along roads. All RRUs are connected to BBU pools through highspeed backhauls. MmW-RRUs and LF-RRUs can exchange information via X3 interfaces. To enhance capacity as well as guarantee transmission reliability, the relatively critical data are carried by reliable sub- $6 \mathrm{GHz}$ bands, while the highvolume service data are moved to broadband mmWave bands. On vehicles, we assume that there are two types of transceivers operating at sub-6 GHz and mmWave bands, respectively. Since in this paper we focus on the handover problems, we only introduce the main function of this network architecture. More design details about the $\mathrm{C} / \mathrm{U}$-plane decoupled network architecture can be found in [5]. 


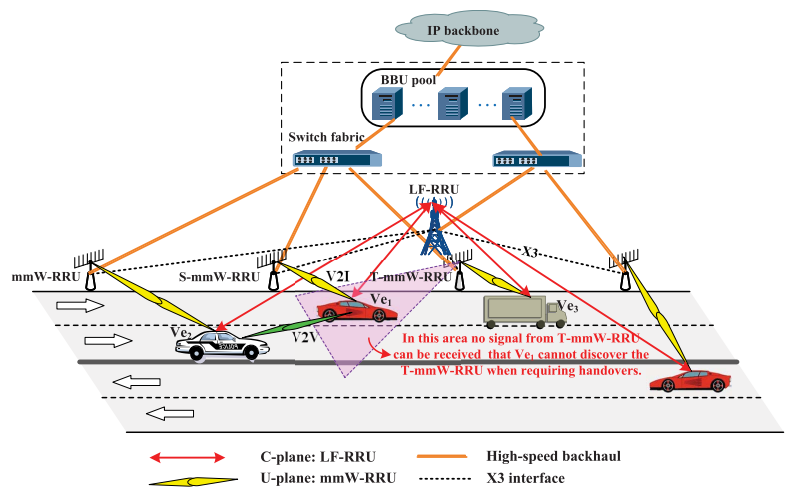

Fig. 1. Network architecture.

\section{B. Handover Problems in mmWave Vehicular Networks}

In mmWave vehicular networks, the coverage blindness of directional beams, the high-mobility of vehicles, and the hard handover respectively cause problems in handovers, i.e., target discovery difficulties, limited handover time and frequent transmission interruptions, which are discussed as follows. Firstly, although to some extent the effects of coverage blindness in mmWave directional communications can be overcome through the integration of sub- $6 \mathrm{GHz}$ and mmWave bands, it is still challenging for the target discovery during V2I handovers. As shown in Fig. 1, $\mathrm{Ve}_{1}$ served by $\mathrm{S}-\mathrm{mmW}$-RRU is moving out of the coverage of S-mmW-RRU and needs to switch to another mmW-RRU. From the perspective of geography, $\mathrm{T}-\mathrm{mmW}$-RRU is the best choice for $\mathrm{Ve}_{1}$ to hand over to. Nevertheless, the active beam of T-mmW-RRU is directed towards $\mathrm{Ve}_{3}$ which is far from $\mathrm{Ve}_{1}$. As a consequence, $\mathrm{Ve}_{1}$ cannot detect any signals from T-mmW-RRU and thus will not choose T-mmW-RRU as a candidate mmW-RRU. From the above analysis, we can observe that the coverage blindness of directional beams makes it challenging for vehicles to discover candidate mmW-RRUs during handovers. In the worst case, handover vehicles even cannot detect any candidate mmW-RRUs and have to reconnect to the network. Moreover, in vehicular networks, the high mobility of vehicles relative to static roadside mmW-RRUs results in less time left for handovers. This situation will get even worse in mmWave communications where the complicated and time-consuming beam training processes are involved in the synchronization of handovers. Finally, the widely adopted hard handover in current wireless networks works in the break-before-make mode, where the cutoff of source connections occurs prior to the establishment of a new one, causing transmission interruptions. While in high-mobility vehicular networks, handovers happen frequently. As a result, in this scenario, hard handovers will cause frequent transmission interruptions, severely degrading the quality of services.

\section{Proposed ML Based Handover Schemes}

To solve the target discovery problem caused by the coverage blindness of mmWave directional beams, a possible way is to pre-activate candidate $\mathrm{mmW}$-RRUs near handover vehicles, where the position information of handover vehicles becomes a key factor. According to the statistical results in [17], CSI

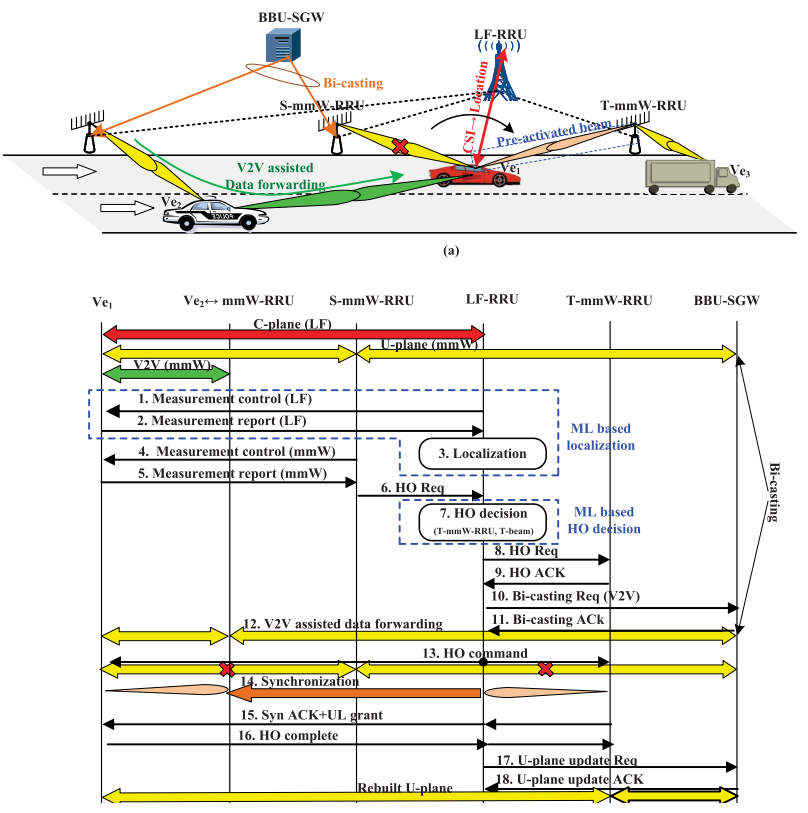

Fig. 2. (a) The ML and V2V assisted soft and fast V2I handover scheme, (b) signaling procedures.

values of multiple sub-carriers exhibit great stability over time at the same position but apparent variability at different positions. Therefore, by combining the powerful ML algorithms and the environment-dependent wireless channels, we can predict vehicles' positions based on the constantly collected CSI in cellular networks without involving extra feedback overheads. In the $\mathrm{C} / \mathrm{U}$-plane decoupled network architecture, with the omni-directional coverage, LF-RRUs can continuously collect CSI from vehicles. Consequently, as shown in Fig.2(a), we utilize the CSI obtained on sub-6 GHz bands as the input feature data of the Kernel-based ML algorithm to estimate vehicles' positions, where the operational details are depicted in Fig. 3. Based on the obtained position information, when a vehicle requests V2I handovers, LF-RRUs will preactivate the candidate mmW-RRUs to sweep beams towards the handover vehicle. According to the measurement reports, LF-RRUs then determine a final target mmW-RRU for vehicles to hand over to.

In high-mobility environments where users rapidly go through handover overlapping areas, there may not exist enough time to conduct handovers. Moreover, complicated beam training processes during handovers in mmWave communications can further aggravate this problem. Nevertheless, in vehicular scenarios, such as highways, the mobility patterns of vehicles on almost linearly paved roads with finite and deterministic corner turns present a strong regularity, which intuitively will also produce some regularity in handover decision-making. Based on this observation, to accelerate handovers, we propose to use historical handover data and the KNN ML algorithm to make predictive handover results, instead of going through complicated target selection and beam training processes. The historical handover data recorded in a defined handover data collection phase include the status information of vehicles when requesting handovers and the corresponding final handover decisions, which are then respec- 


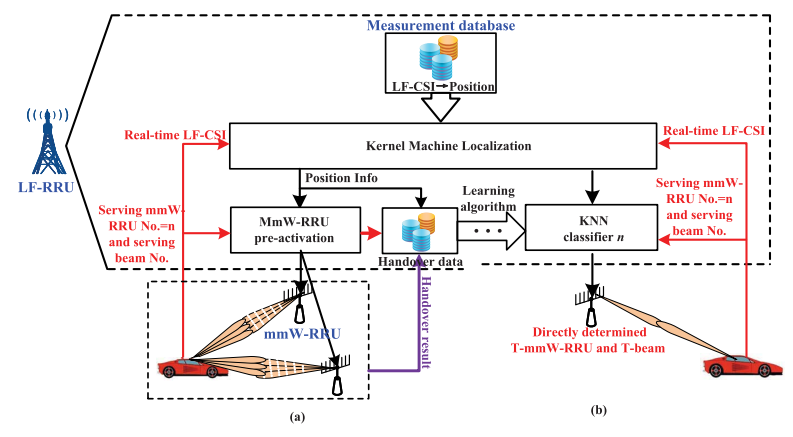

Fig. 3. (a) The handover data collection phase, (b) the fast handover phase.

tively used as the input feature data and output label data of the KNN ML algorithm to exploit their internal relationships. Afterwards, in the fast handover phase, as shown in Fig. 2(a), final handover decisions are directly determined based on the status information of handover vehicles without involving the complicated and time-consuming target selection and beam training processes, thereby accelerating V2I handovers. In Fig. 2(b), the signaling procedures of the proposed V2I handover scheme are designed, in which Steps 1-3 present the CSI based positioning, Step 7 corresponds to the handover decision-making based on a KNN ML algorithm and other steps related to $\mathrm{V} 2 \mathrm{~V}$ assisted data forwarding are discussed later.

The type of handovers used in current wireless communication networks is hard handover, where users have to cut off current connections with source base stations (BSs) before establishing new connections with target BSs, potentially causing temporary transmission interruptions. In future intelligent vehicles, besides on outside roofs, mmWave transceivers can also be installed on the four sides of vehicles to gain more opportunities of building $\mathrm{V} 2 \mathrm{~V}$ links to achieve a fully interconnected vehicular network. Such massive available V2V links may provide a possible solution to transmission interruptions during V2I hard handovers. In addition, since vehicles moving along the same direction have low relative speeds the established V2V links are more stable compared to V2I links between static mmW-RRUs and traveling vehicles. Based on this observation, we propose to use V2V links to forward data for V2I links that experience handovers. With recorded position information of vehicles, it is reasonable to assume that through some simple operations, LF-RRUs can infer the speed and direction of vehicles. Therefore, the assisting V2V links for data forwarding can be purposefully established between the vehicle requesting V2I handovers and another vehicle moving along the same direction with low relative speed. As shown in Fig. 2(b), Steps 8-20 correspond to the designed V2V assisted V2I handover signaling. After the T-mmW-RRU acknowledges the handover request, LF-RRUs will ask the BBU-SGW to duplicate and bi-cast the service data of $V_{1}$ to both the S-mmW-RRU and the mmW-RRU that the $\mathrm{V} 2 \mathrm{~V}$ partner $\mathrm{Ve}_{2}$ is currently attaching to, which equivalently establishes an additional U-plane for $\mathrm{Ve}_{1}$ through V2V links. After a handover command in Step 13 is received, $\mathrm{Ve}_{1}$ will cut off the original connection with S-mmW-RRU, and then follow the handover decision obtained through the
KNN ML algorithm in Step 7 to directly align beams with the determined target mmW-RRU. Following the synchronization, the air interface of the U-plane connection between $\mathrm{Ve}_{1}$ and the network will be updated and transferred to T-mmW-RRU. Finally, BBU-SGW will exit data bi-casting and the whole handover process is completed.

\section{Mathematical Models}

In this section, mathematical models to evaluate the ML based positioning and fast handover decision-making schemes and the V2V assisted V2I handover mechanism are developed. In Fig. 3, the procedures of the proposed ML based positioning and fast handover decision-making schemes are shown, in which Fig. 3(a) and Fig.3 (b) show the handover data collection phase and the ML based fast handover phase, respectively. Based on a wireless channel fingerprint database which is pre-established through field measurements similarly to [24], we can obtain the relationship between the CSI and the position information via the Kernel-based ML algorithm. According to vehicles' positions, LF-RRUs pre-activate neighboring mmW-RRUs to sweep beams towards the vehicles requesting handovers. Handover vehicles then feedback the measurement reports of signal quality, which are used by LFRRUs as the basis to make the final handover decision. During the handover data collection phase, the status information of handover vehicles and the corresponding handover decisions are recorded and then used as training data to build KNN classifiers in LF-RRUs. To simplify the system implementation, in LF-RRUs, a dedicated KNN classifier is allocated for every $\mathrm{mmW}$-RRU to provide handover decision-making functions for vehicles being served by this specific mmWRRU. According to the index of the source mmW-RRU which is contained in the status information fed back by a handover vehicle, LF-RRUs locate the corresponding KNN classifier and input the rest status information of the vehicle into this KNN classifier. The output handover decision will directly guide the handover vehicle and the target mmW-RRU to align their beams without involving complicated and time-consuming target selection and beam training processes. Overall, the handover data collection phase aims at getting handover related data by using the conventional handover protocols, while the fast handover phase aims at using the KNN ML algorithm to exploit the internal relationship between these recorded handover data and then using the obtained KNN classifiers to give fast handover decisions.

\section{A. The Kernel-Based ML Positioning}

The Kernel-based ML positioning consists of two components, i.e., the off-line training and the on-line prediction. Before the off-line training, CSIs at different positions are sampled through field measurements, similar to [24], and form a wireless channel fingerprint database. In [17], it has been shown that the amplitude information contained in CSI is an effective feature in predicting users' positions. To simplify the analysis, in this paper, the highway scenario with much cleaner V2I signal propagation environments is taken into 
account, where wireless channels can be approximated as lineof-sight (LOS) channels with large coherence bandwidth [25]. Therefore, a large spectrum granularity is used to at most remove correlations of the amplitude information between adjacent samples, reducing the dimensionality of input feature data. Suppose that the whole spectrum is divided into $M$ chunks and each of them $c_{i}$ provides an amplitude element of the input feature data. The observed input CSI feature vector can be expressed as

$$
\mathbf{h}_{\mathbf{p}_{i}}=\left[h_{\mathbf{p}_{i}, c_{1}}, \ldots, h_{\mathbf{p}_{i}, c_{M}}\right]^{T}
$$

where $\mathbf{p}$ represents the coordinates of the position where this CSI sample comes from. $h_{\mathbf{p}_{i}, c_{j}}$ is the amplitude of chunk $j$ at position $\mathbf{p}_{i}$, which follows the Rician distribution under the assumption of LOS channels. Suppose the total number of samples is $N$ and a $D$-dimensional space is considered. The coordinates of the $i$ th position, where $i=1,2, \ldots, N$, can be written as

$$
\mathbf{p}_{i}=\left[p_{i, 1}, \ldots, p_{i, D}\right]^{T} \in \mathbb{R}^{D} .
$$

The off-line training phase aims to find the relationship function $\psi$ that can map CSI feature vectors to the corresponding position vectors with least errors. For simplicity, we divide the pending relationship function into $D$ sub-functions, i.e., $\psi(\cdot)=\left[\psi_{1}(\cdot), \ldots, \psi_{D}(\cdot)\right]$, where $\psi_{d}: \mathbb{R}^{M} \mapsto \mathbb{R}$ outputs the prediction of the $d$ th dimension value $p_{i, d}$ of $\mathbf{p}_{i}=\left[p_{i, 1}, \ldots, p_{i, D}\right]^{T}$ with a given $\mathbf{h}_{\mathbf{p}_{i}}$. The training process of $\psi_{d}(\cdot)$ to approach the real relationship between CSI samples and the corresponding positions with least errors can be formulated as [26]

$$
\min \frac{1}{N} \sum_{i=1}^{N}\left(p_{i, d}-\psi_{d}\left(\mathbf{h}_{\mathbf{p}_{i}}\right)\right)^{2}+\varphi\left\|\psi_{d}\right\|_{H}^{2}
$$

where $\varphi$ is a regularization parameter to control the tradeoff between the training error and the complexity of the solution. $H$ denotes the reproducing Kernel Hilbert space with inner product $\langle\cdot, \cdot\rangle_{H}$.

Based on the results of [27], the optimal function of $\psi_{d}(\cdot)$ is in the form of

$$
\psi_{d}(\cdot)=\sum_{j=1}^{N} a_{d, j} \kappa\left\langle\cdot, \mathbf{h}_{\mathbf{p}_{j}}\right\rangle .
$$

Let

$$
\begin{aligned}
\mathbf{a}_{d} & =\left[a_{d, 1}, \ldots, a_{d, N}\right]^{T}, \\
\mathbf{K} & =\left(\kappa\left\langle\mathbf{h}_{\mathbf{p}_{i}}, \mathbf{h}_{\mathbf{p}_{j}}\right\rangle\right)_{N \times N},
\end{aligned}
$$

and

$$
\mathbf{y}=\left[p_{1, d}, \ldots, p_{N, d}\right]^{T} .
$$

We can rewrite expression (3) as

$$
\min \frac{1}{N}\left(\mathbf{y}-\mathbf{K} \cdot \mathbf{a}_{d}\right)^{T}\left(\mathbf{y}-\mathbf{K} \cdot \mathbf{a}_{d}\right)+\eta \mathbf{a}_{d}^{T} \mathbf{a}_{d} .
$$

By taking the derivative of (8) with respect to $\mathbf{a}_{d}$ and setting the result to 0 , we can obtain

$$
\mathbf{a}_{d}=\mathbf{K}^{T} \mathbf{y}\left(\mathbf{K}^{T} \mathbf{K}+\eta N \mathbf{I}\right)^{-1} .
$$

Finally, substituting the results of (9) into (4), we can get the Kernel-based ML positioning model.

In this paper, we consider the Gaussian Kernel with the expression of

$$
\kappa\left\langle\mathbf{h}_{\mathbf{p}_{j}}, \mathbf{h}_{\mathbf{p}_{i}}\right\rangle=\exp \left(\frac{-\left\|\mathbf{h}_{\mathbf{p}_{j}}-\mathbf{h}_{\mathbf{p}_{i}}\right\|^{2}}{2 \sigma^{2}}\right) .
$$

where $\sigma$ is the bandwidth parameter of Gaussian Kernel. Equivalently, the final obtained position prediction model is approximated by a weighted superposition of Gaussian functions. The weight value of each Gaussian function is derived by minimizing the estimation error on the training set.

Suppose there are $Q$ mmW-RRUs under an LF-RRU and they are sequentially numbered as $1,2, \ldots, Q$. Based on the estimated position information of handover vehicles, mmW-RRUs that have a distance lower than a threshold $d_{t h}$ to the handover vehicle can be selected as target candidates and pre-activated, that is

$$
Q_{s}=\underset{i=1, \ldots, Q}{\arg }\left(\left\|\widehat{\mathbf{p}}_{j}-\mathbf{p}_{m m W, i}\right\| \leq d_{t h}\right)
$$

where $\mathbf{p}_{m m W, i}$ is the coordinate of mmW-RRUs.

\section{B. The KNN ML Handover Decision-Makings}

In vehicular scenarios, mobility patterns of vehicles on almost linearly paved roads with finite and deterministic corner turns show a strong regularity, which intuitively will also lead to some regularity in handovers. Based on this observation, we propose a KNN ML based handover scheme, which consists of two parts, i.e., the handover data collection phase and the fast handover phase. In the handover data collection phase, LF-RRUs record the status information of vehicles when requesting handovers (including the position information, the serving mmW-RRU's index and the serving beams' indices of both handover vehicles and serving mmWRRUs at that time) and the corresponding final handover decisions (including the selected target mmW-RRU's index and the target beams' indices of both handover vehicles and the selected target mmW-RRUs). In this scheme, for every subordinate mmW-RRU, LF-RRUs will allocate a dedicated $\mathrm{KNN}$ classifier to provide fast handover decision-making functions for vehicles served by this mmW-RRU. Since $Q$ mmW-RRUs are within the coverage of an LF-RRU, $Q$ KNN classifiers are configured in the LF-RRU with each of them corresponding to a certain mmW-RRU. Without loss of generality, we sequentially number the $Q \mathrm{KNN}$ classifiers and then the status information vector of vehicles can be expressed as

$$
\mathbf{u}_{i}=\left[\widehat{\mathbf{p}}_{i}^{T}, n_{S, i}, n_{S B, i}^{S_{i}}, n_{V, i}^{S_{i}}\right]
$$

where $\widehat{\mathbf{p}}_{i}$ represents the position information. The serving mmW-RRU' index of handover vehicles is denoted by $n_{S, i}$ with $n_{S, i}=1,2, \ldots, Q \cdot n_{S B, i}^{S_{i}}$ and $n_{V e, i}^{S_{i}}$ are the serving beams' indices of the serving mmW-RRU and the handover vehicle, respectively, with $n_{S B, i}^{S_{i}}=1,2, \ldots, L_{B}$ and $n_{V, i}^{S_{i}}=1,2, \ldots, L_{V}$ where $L_{B}$ and $L_{V}$ separately denotes the total number of beams at mmW-RRUs and vehicles. 
The handover decisions corresponding to the status information $\mathbf{u}_{i}$ can be given in the form of vectors as

$$
\mathbf{w}_{i}=\left[n_{T, i}, n_{T B, i}^{T_{i}}, n_{V, i}^{T_{i}}\right]
$$

where $n_{T, i}=1,2, \ldots, Q, n_{T B, i}^{T_{i}}=1,2, \ldots, L_{B}$, and $n_{V, i}^{T_{i}}=1,2, \ldots, L_{V}$ represent the selected target mmW-RRU's index, the target beams' indices of the selected target $\mathrm{mmW}$-RRU and the handover vehicle, respectively.

For a given mmW-RRU, after getting sufficient handover data in the handover data collection phase, the status information of handover vehicles acting as the input feature data and the corresponding handover decisions acting as the classification labels will be used to build the KNN classifier. In the next fast handover phase, once inputting the status information of handover vehicles into the classifier, for every classification, the $K$ nearest neighbors in the database will be selected and the classification with the smallest superposition results of the $K$ shortest distance will be determined as the output. In this paper, the metric of Euclidian distance is adopted to measure the similarity of two input feature vectors, that is [28]

$$
d\left(\mathbf{u}_{i}, \mathbf{u}_{j}\right)=\left(\mathbf{u}_{i}-\mathbf{u}_{j}\right) \cdot\left(\mathbf{u}_{i}-\mathbf{u}_{j}\right)^{T} .
$$

In vehicular environments, the roads are paved almost linearly with finite and deterministic corner turns, which implies that for a given mmW-RRU, handovers will mostly take place at some specific beams such as those near turning corners. In view of this point, it will be an effective and easy way to lower the prediction error of the KNN ML algorithm by maximizing the numbering distance between these beams where handovers often occur. Note that here we only consider the inter-mmW-RRU handovers, instead of intra-mmW-RRU beam switchings which usually take place between two adjacent beams and do not need predictions. Therefore, we assume that handovers will mostly take place at some specific beams. Suppose for an mmW-RRU, handovers mostly happen at $M$ beams. Before building the KNN classifier, the numbering of these $M$ beams can be re-numbered as

$$
\left(m_{1}, \ldots, m_{M}\right)=\max _{m_{i}, m_{j}=1, \ldots, L}\left(\min _{i, j=1, \ldots, M}\left|m_{i}-m_{j}\right|\right) .
$$

Actually, in the KNN ML algorithm, there is no offline training. All computations are conducted in the on-line part, where the Euclidian distances between the input and stored data are calculated and compared for classifications. As aforementioned, to reduce the computation complexity, in the proposed scheme, every mmW-RRU is configured with a dedicated KNN classifier to deal with the handover decisions. In an mmW-RRU, handovers usually happen at some edge beams or beams near turning corners. In addition, the handover overlapping areas of beams are very narrow. Therefore, the stored handover data will not be in a large volume. In other words, the computation burden of using the KNN ML algorithm in this scenario will not be that heavy. For instance, we assume that in a given mmW-RRU all handovers happen on 4 beams. Suppose the coverage diameter of a beam is $5 \mathrm{~m}$, and 50 typical handover samples in the coverage are stored. Then, the total number of main computations of the Euclidian distance between a new input and the database is $50 \times 4=200$. For simplicity, we compare the superposition results of $K$ shortest distances and select the classification label with the smallest result as the final output. Nevertheless, other methods, such as weighted KNN algorithms, can also be applied to this scheme.

\section{V2V Assisted Data Forwarding}

As aforementioned, the commonly used hard handover in current wireless communication systems can cause temporary transmission interruptions, which severely degrades the transmission reliability. To address this issue, we propose to use V2V links that can be massively attainable in future vehicular networks to forward data for vehicles that are executing V2I handovers. In this section, we will theoretically analyze the connectivity probability, the average signal to noise ratio (SNR) and the interruption probability of V2V links when being used to forward data during V2I handovers.

Connectivity probability is the probability that at least one partner vehicle can be found at the $\mathrm{V} 2 \mathrm{~V}$ reachable distance with respect to the considered handover vehicle. To guarantee the V2V connection stability, only vehicles moving along the same direction as the handover vehicles are elected as partners to assist V2I handovers. Suppose there are $Z$ lanes in the same direction sequentially numbered as $i=0,1, \ldots, Z-1$ and only one vehicle can cross a lane at any given time. Then, the number of $\mathrm{V} 2 \mathrm{~V}$ partner vehicles on different lanes can be assumed to identically and independently follow a Poisson Process with intensity $\lambda$. We use $w_{s}$ and $r_{\max }$ to denote the width of lanes and the maximum communication distance of V2V links, respectively. Assuming that the handover vehicle is on lane 0 , the probability that there are $j$ partner vehicles on lane $i$ around the handover vehicle within the distance of $r$ can be expressed as [29]

$$
P_{L, i}(r, j)=\frac{\left(2 \lambda \sqrt{r^{2}-\left(i \cdot w_{s}\right)^{2}}\right)^{j}}{j !} e^{-2 \lambda \sqrt{r^{2}-\left(i \cdot w_{s}\right)^{2}}} .
$$

Then, the connectivity probability, which can be equivalently interpreted as the probability that there is at least one vehicle within the maximum communication distance of $\mathrm{V} 2 \mathrm{~V}$ links, can be given by [30]

$$
\begin{aligned}
P_{\text {contact }} & =1-\Pi_{i=0}^{Z-1} P_{L, i}\left(r_{\max }, 0\right) \\
& =1-e^{-2 \lambda \sum_{i=0}^{Z-1} \sqrt{r_{\max }^{2}-i^{2} w_{s}^{2}}} .
\end{aligned}
$$

Average SNR can reflect the capacity of V2V links to a certain extent. For simplicity, in this paper, in order to obtain high-quality V2V assisting links, it is assumed that the nearest partner vehicle with respect to the handover vehicle is elected to establish relatively high-performance LOS connections. According to [31], the probability that the nearest partner vehicle is at least $r$ away from the handover vehicle is equivalent to the one that within $r$ there is no vehicle, i.e.,

$$
\begin{aligned}
P(d a \geq r) & =\Pi_{i=0}^{Z-1} P_{L, i}(r, 0) \\
& =e^{-2 \lambda \sum_{i=0}^{Z-1} \sqrt{r^{2}-i^{2} w_{s}^{2}}} .
\end{aligned}
$$


where $d a$ denotes the distance between the two cooperative vehicles. Subsequently,

$$
\begin{aligned}
P(d a \leq r) & =1-P(d a \geq r) \\
& =1-e^{-2 \lambda \sum_{i=0}^{Z-1} \sqrt{r^{2}-i^{2} w_{s}^{2}}} .
\end{aligned}
$$

By taking the derivation of (18) with respect to $r$, the probability density function of the propagation distance of $\mathrm{V} 2 \mathrm{~V}$ links is obtained as

$$
f(r)=2 \lambda e^{-2 \lambda \sum_{i=0}^{Z-1} \sqrt{r^{2}-i^{2} w_{s}^{2}}} \sum_{i=0}^{Z-1} \frac{r}{\sqrt{r^{2}-i^{2} w_{s}^{2}}} .
$$

Based on (19), the average SNR of V2V links when forwarding data for V2I handovers can be expressed as

$$
\overline{S N R}=\int_{(Z-1) w_{s}}^{r_{\max }} \frac{P_{T} G_{R} G_{T}}{\sigma^{2}} A r^{-\gamma} f(r) d r
$$

where $P_{T}, G_{T}, G_{R}, \sigma^{2}, A$ and $\gamma$ denotes the transmit power, the transmitter beam gain, the receiver beam gain, the Gaussian white noise power, the constant part of path loss and the exponential coefficient of path loss, respectively.

V2V interruption probability, which can capture the connection stability of $\mathrm{V} 2 \mathrm{~V}$ links, is defined as the probability that the holding time of $\mathrm{V} 2 \mathrm{~V}$ links is shorter than the required time of V2I handovers. Without loss of generality, we assume that the velocity of a handover vehicle, denoted by $v_{1}$, is lower than that of a partner vehicle denoted by $v_{2}$. With a V2V propagation distance of $r$, there are two situations, i.e., the partner vehicle is in the head or the rear of the handover vehicle. Considering the worst case that the partner vehicle with higher velocity is in the head of the handover vehicle, the holding time of this $\mathrm{V} 2 \mathrm{~V}$ link when the partner vehicle is on lane $i$ can be given by

$$
T_{w, i}(r)=\frac{\sqrt{r_{\max }^{2}-i^{2} w_{s}^{2}}-\sqrt{r^{2}-i^{2} w_{s}^{2}}}{\left|v_{1}-v_{2}\right|} .
$$

Denoting the required handover time as $T_{H O}$, the interruption probability of V2V links during V2I handovers can be further expressed as

$$
\begin{aligned}
& P_{\text {out }, i}\left(T_{w, i}(r)<T_{H O}\right) \\
& =P\left(r>\sqrt{\left(\sqrt{r_{\max }^{2}-i^{2} w_{s}^{2}}-\left|v_{1}-v_{2}\right| T_{H O}\right)^{2}+i^{2} w_{s}^{2}}\right) .
\end{aligned}
$$

Based on the above analysis, the probability of the event that the partner vehicle on lane $i$ is within the distance of $r$ away from the handover vehicle can be similarly derived as

$$
f_{i}(r)=\frac{2 \lambda r}{\sqrt{r^{2}-i^{2} w_{s}^{2}}} e^{-2 \lambda \sqrt{r^{2}-i^{2} w_{s}^{2}}}
$$

Let $\varepsilon=\sqrt{\left(\sqrt{r_{\max }^{2}-i^{2} w_{s}^{2}}-\left|v_{1}-v_{2}\right| T_{H O}\right)^{2}+i^{2} w_{s}^{2}}$, then expression (22) can be further written as

$$
P_{\text {out }, i}\left(T_{w, i}(r)<T_{H O}\right)=\int_{\varepsilon}^{r_{\max }} f_{i}(r) d r .
$$

\section{Vi. Simulation Results and Discussions}

In this section, simulation results using MATLAB are presented to validate the feasibility of the proposed handover scheme. It should be noted that the main aim of this paper is to use ML algorithms to solve handover problems in mmWave vehicular networks, instead of performance improvements of ML algorithms themselves. Therefore, we take two typical ML algorithms, i.e., Kernel-based and KNN ML algorithms, as examples, to illustrate our proposed scheme. Of course, other more powerful ML algorithms can also be incorporated into our schematic design.

\section{A. Performance of the Kernel-Based ML Positioning}

In this subsection, before the performance analysis of the Kernel-based ML positioning scheme, the wireless channel models to generate the environment-dependent CSI data of sub-6 GHz bands are introduced. For simplicity, in this paper, the highway scenario with much cleaner V2I signal propagation environments is taken into account, where the wireless channels exhibit the feature of near LOS and follow the Rician distribution [32]. Based on this assumption, at position $\mathbf{p}_{i}$, the distribution of the amplitude of chunk $c_{j}$ can be expressed as

$$
\begin{aligned}
f_{R}(x)=\frac{2\left(K_{L}+1\right) x}{\overline{x_{\mathbf{p}_{i, c_{j}}}}} \exp & \left(-K_{L}-\frac{\left(K_{L}+1\right) x^{2}}{\overline{x_{\mathbf{p}_{i, c_{j}}}}}\right) \\
& \times I_{0}\left(2 x \sqrt{\frac{K_{L}\left(K_{L}+1\right)}{\overline{x_{\mathbf{p}_{i, c_{j}}}}}}\right)
\end{aligned}
$$

where we use $K_{L}$ to represent the $K$ factor of LOS channels so as to differentiate from the parameter $K$ of the KNN ML algorithm. $\overline{x_{\mathbf{p}_{i}}}$ is the total received energy, expressed as

$$
\overline{x_{\mathbf{p}_{i}}}=10 \frac{P_{L F, T}-P L\left(\left\|\mathbf{p}_{i}-\mathbf{p}_{L F-R R U}\right\|, c_{j}\right)+\xi}{10},
$$

where $P_{L F, T}$ is the transmit power of LF-RRUs in the unit of $\mathrm{dBm}$. $\mathbf{p}_{L F-R R U}$ denotes the coordinates of the LF-RRU's position. $P L\left(\left\|\mathbf{p}_{i}-\mathbf{p}_{L F-R R U}\right\|, c_{j}\right)$ stands for the path loss in the unit of $\mathrm{dB}$ under a signal propagation distance of $\left\|\mathbf{p}_{i}-\mathbf{p}_{L F-R R U}\right\|$ and an operating frequency center of $c_{j}$. $\xi$ represents the shadow fading in the unit of $\mathrm{dB}$ which follows the $\log$ normal distribution with zero mean and $\sigma_{\xi}^{2}$ variance. According to [33], the Rician-distributed amplitude of signals can be generated by adding an LOS component into a Rayleigh-distributed component which can be easily obtained through MATLAB.

Actually, in addition to path loss, based on the field test results measured in [34], the $K$ factor of Rician-distributed LOS wireless channels is also environment-dependent, which can be approximated as

$$
\begin{aligned}
K_{L}\left(\left\|\mathbf{p}_{i}-\mathbf{p}_{L F-R R U}\right\|\right) \\
=8.53-4.39 \log _{10}\left(\frac{\left\|\mathbf{p}_{i}-\mathbf{p}_{L F-R R U}\right\|}{d_{0}}\right)
\end{aligned}
$$

where $d_{0}=1 \mathrm{~km}$ is a reference distance.

Based on the above results, two groups of data are generated according to the parameter settings in Table I [35], [36], 
TABLE I

PARAMETER SETting [35]

\begin{tabular}{|c|c|}
\hline Parameters & Values \\
\hline Coverage radius of LF-RRUs & $500 \mathrm{~m}$ \\
Transmit power of LF-RRUs & $43 \mathrm{dBm}$ \\
Transmit power of mmW-RRUs & $30 \mathrm{dBm}$ \\
Transmit power of vehicles & $30 \mathrm{dBm}$ \\
Frequency center of LF-RRUs & $2 \mathrm{GHz}$ \\
Frequency center of mmW-RRUs & $30 \mathrm{GHz}$ \\
Bandwidth of LF-RRUs & $20 \mathrm{MHz}$ \\
Bandwidth of mmW-RRUs & $1 \mathrm{GHz}$ \\
Distance between LF-RRUs and roads & $30 \mathrm{~m}$ \\
Distance between mmW-RRUs and roads & $5 \mathrm{~m}$ \\
Path loss models of LF/mmWave bands & Free space \\
mmWave transmitter/receiver antenna gain & $10 \mathrm{~dB}$ \\
Width of lanes & $4 \mathrm{~m}$ \\
Number of handover beams in mmW-RRUs & 4 \\
Variance of shadowing & $6 \mathrm{~dB}$ \\
Total number of beams in mmW-RRUs & 64 \\
Handover time consumption & $0.5 \mathrm{~s}$ \\
Coverage radius of beam handover area & $5 \mathrm{~m}$ \\
Sampling interval in $x$-dimension & $5 \mathrm{~m}$ \\
Sampling interval in $y$-dimension & $4 \mathrm{~m}$ \\
Velocity of handover vehicles & $30 \mathrm{~m} / \mathrm{s}$ \\
Velocity of partner vehicles & $20 \mathrm{~m} / \mathrm{s}$ \\
Number of samples used in KNN ML algorithm & 50 \\
\hline \multicolumn{2}{|c|}{} \\
\hline
\end{tabular}

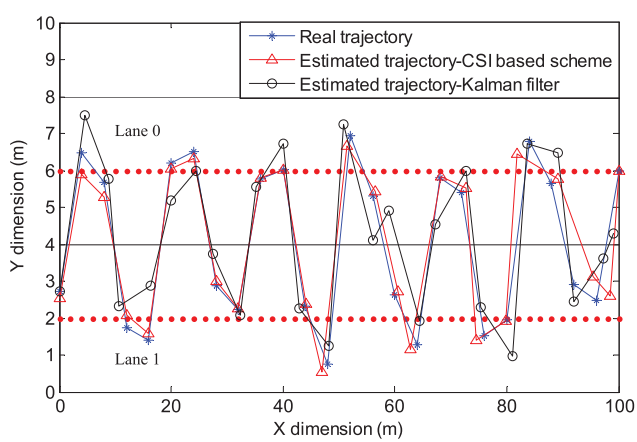

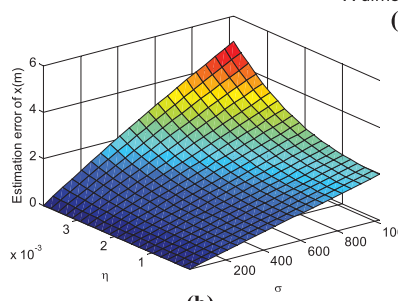

(b) (a)

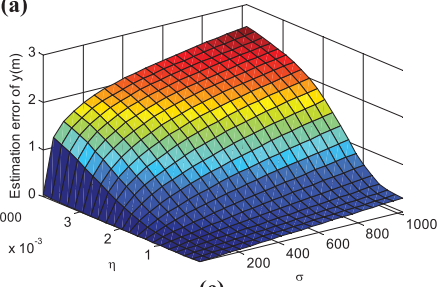

(c)
Fig. 4. (a) Trajectory estimation, (b) estimation errors of $x$-dimension, (c) estimation errors of $y$-dimension.

in which one group is used to train the off-line positioning model, and the other is used for the on-line testing to evaluate the estimation error. Without loss of generality, the two-dimensional positioning is considered, i.e., $D=2$. In Fig. 4(a), an example of the trajectory estimations under different schemes are shown. For the proposed scheme, the simulation parameters are set to $\sigma=4$ and $\eta=2^{-18}$, under which the average estimated errors of the $x$-dimension and $y$-dimension are $0.62 \mathrm{~m}$ and $0.17 \mathrm{~m}$, respectively. Besides, in Fig.4(a), the Kalman filter based positioning scheme is employed to compare the positioning performance between different methods, in which we set both the measurement error variances of $x$-dimension and $y$-dimension to $1 \mathrm{~m}$ [37]. The result shows that after the Kalman filter process, the final estimation errors of the $x$-dimension and $y$-dimension are reduced to $0.63 \mathrm{~m}$ and $0.75 \mathrm{~m}$, respectively. Obviously, the CSI based method can achieve the same or even better positioning accuracy compared with the conventional Kalman filter based positioning method. More than that, in the Kalman filter scheme, vehicles need to periodically feed back movement related information to help the network to figure out more accurate trajectories. Suppose the position measurements of vehicles are obtained through the mostly used Global Positioning System (GPS) whose information update rate $r_{G P S}$ is typically $1 \sim 10 \mathrm{~Hz}$, i.e., updating positioning results every 0.1 1s [37]. By denoting the volume of quantized position information as $\omega_{G P S}$ bits, the feedback overhead of the Kalman filter based positioning scheme can be expressed as $r_{G P S} \times \omega_{G P S}$ bits/s. Instead, in the proposed scheme, the key data used for trajectory estimations are CSI, which are constantly collected by wireless communication systems themselves anyhow, thereby without causing extra feedback overhead. In addition, the CSI update period of wireless communication systems is usually as short as several milliseconds, much shorter than the positioning information update period of GPS, thereby enabling more accurate and timely tracking of high-mobility vehicles. To give a comprehensive evaluation of the proposed scheme, in Fig.4 (b) and (c), the estimation errors of $x$-dimension and $y$-dimension under different parameter settings are shown, respectively. Considering the practical vehicular scenarios that one lane is usually occupied by one vehicle, the sampling interval of $y$-dimension is set the same with the width of the lane, while the sampling interval of $x$-dimension is set larger to reduce the sample data volume. As a result, under the same parameter setting, the estimation errors of $x$-dimension and $y$-dimension are different. In both Fig. 4(b) and (c), the estimation error increases with increasing $\sigma$, while the situation is more complicated for the parameter $\eta$. Consequently, in practical applications, the parameter settings should be carefully adjusted to best adapt to different scenarios. It should be noted that the main aim of this paper is to use the obtained position estimation results to solve the target discovery problem. Hence, we do not provide deep analysis results about trajectory estimation algorithms themselves. More details about the trajectory tracking algorithms can be found in [15], [17], [26].

Next, Fig. 5 shows a simple analysis result of the target discovery rate under the two cases, i.e., with and without position information for target pre-activation. In this simulation, 4 antenna arrays are assumed in an mmW-RRUs, and every antenna array has a 0.5 probability to be busy at the time when a vehicle is trying to hand over to this mmW-RRU. For the case without pre-activation, target mmW-RRUs can only depend on beam sweeping to discover potential access/handoverrequesting vehicles. For vehicles in need of handovers, if the sweeping angle space of the target mmW-RRU dose not cover them, this mmW-RRU is missed out. For simplicity, we assume a $30^{\circ}$ effective angle space to cover a handover vehicle, i.e., the probability that an available antenna array can discover a vehicle is 1/12. Nevertheless, for the case using position information to pre-activate target mmW-RRUs, as long as there are available antenna arrays in an mmW-RRU, the handover vehicle can be discovered by sweeping beams towards its direction, thereby improving the target discover rate. 


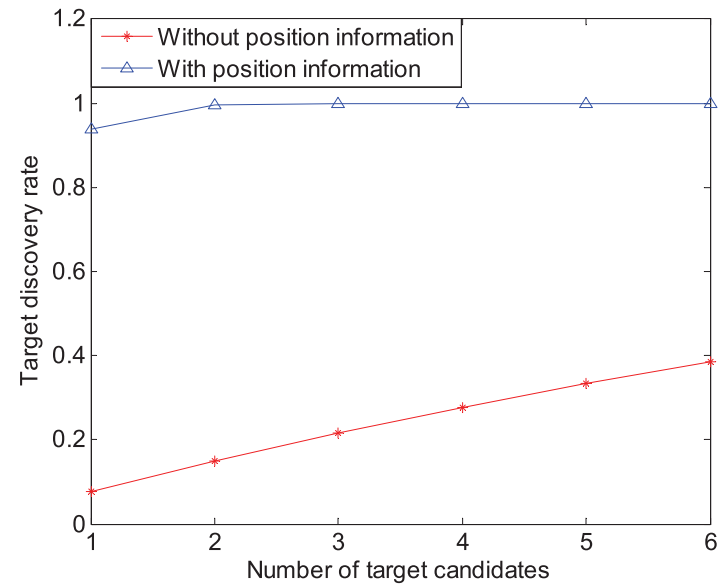

Fig. 5. Target discover rate comparison.

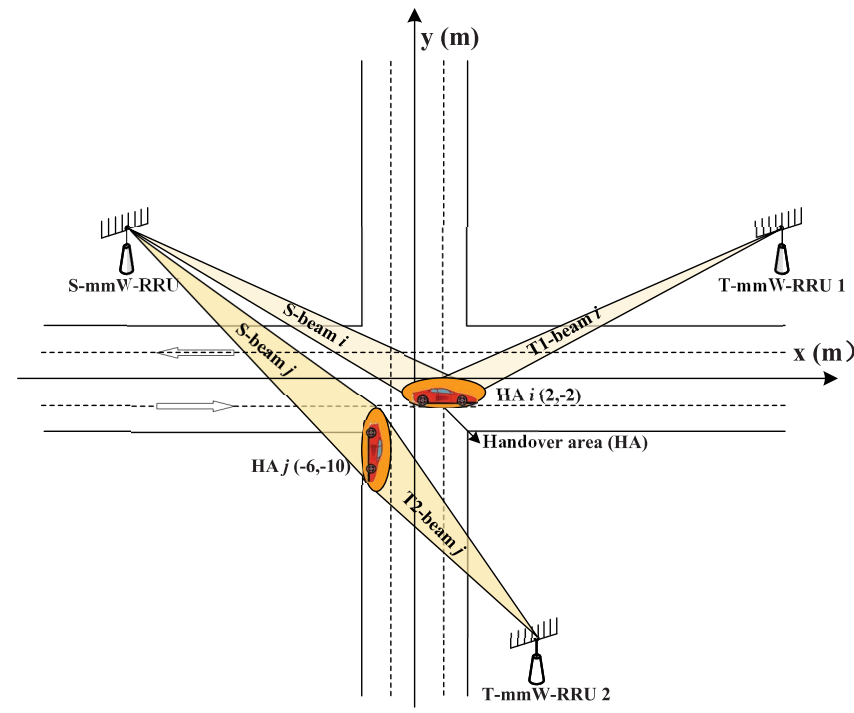

Fig. 6. The simulation scenario of the KNN ML handover decision-making scheme.

\section{B. Performance of the KNN ML Handover Decision-Making}

To evaluate the performance of the proposed fast handover scheme, we take the worst case, which usually happens at intersections, as a case study, where two handover areas of an $\mathrm{mmW}$-RRU are close to each other causing severer confusions in handover decision-makings. The simulation scenario is shown in Fig. 6, where HA $i$ and HA $j$ are two adjacent handover spots of S-mmW-RRU at an intersection. When vehicles keep straight, a handover from S-beam $i$ to T1-beam $i$ should be triggered in HA $i$. If vehicles turn right, a handover from S-beam $j$ to T2-beam $j$ should be triggered in HA $j$. Since at a size-limited intersection two handover areas are close to each other in geography, it is hard to distinguish these two cases during handover decision-makings, thereby easily causing errors. In the simulation, the coordinates of HA $i$ and HA $j$ centers are set to $(2,-2)$ and $(-6,-10)$, respectively. Other simulation parameter values are as listed in Table I.

In Fig. 7, the estimation error rates of the KNN ML handover decision-makings are shown. As aforementioned, in the proposed scheme, to avoid the handover confusions (such as the situation shown in Fig.6), before using the

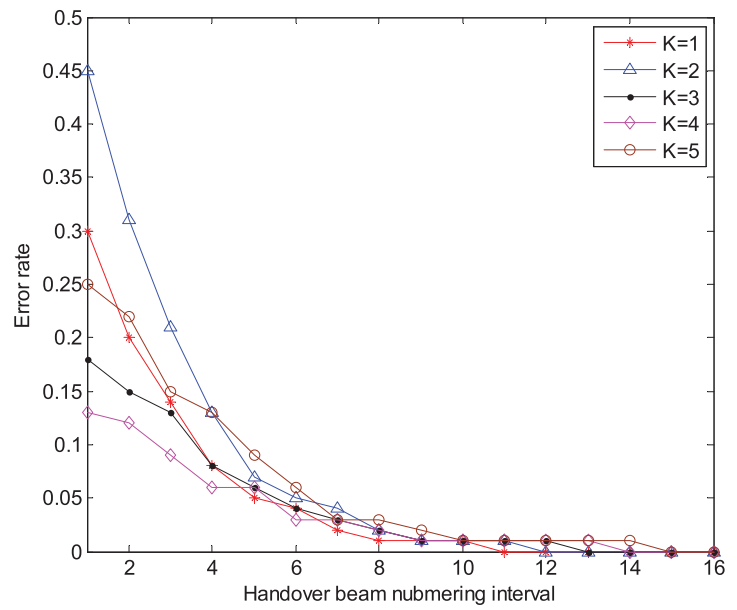

Fig. 7. Estimation error rate of the KNN ML handover decision-making scheme.

TABLE II

Time Consumption of Handover Decision-Making Schemes

\begin{tabular}{|c|c|}
\hline Conventional beam-training based scheme & Time $(\mathrm{ms})$ \\
\hline$L_{B} \times L_{V}=64 \times 64$ & 40.96 \\
$L_{B} \times L_{V}=64 \times 32$ & 20.48 \\
$L_{B} \times L_{V}=64 \times 20$ & 12.80 \\
\hline KNN ML algorithm based scheme & Time $(\mathrm{ms})$ \\
\hline $\mathrm{K}=2$ & 1.95 \\
$\mathrm{~K}=3$ & 2.17 \\
$\mathrm{~K}=4$ & 2.40 \\
\hline
\end{tabular}

collected handover data to build KNN classifiers, the beams where handovers frequently take place are renumbered to maximize the numbering distance. As shown in Fig.7, with increasing handover beam numbering distance, the estimation error rates decrease significantly, and even get close to 0 under sufficient large beam numbering distances. Besides, Fig. 7 also shows the results under different numbers of selected neighbors of the KNN algorithm. Under the current parameter settings, the case of $K=4$ presents the lowest estimation error rate. Notice that the main aim of this paper is to use ML algorithms to accelerate handovers, rather than to improve algorithms themselves. Therefore, we take the simple KNN algorithm as a case study. Nevertheless, other more powerful ML algorithms can also be incorporated here. To explicitly show that the proposed scheme can accelerate handovers, in Table II, the time consumption of handover decision-making processes in different schemes is compared. In the conventional beam-training based handover scheme, after the target mmW-RRU is determined, beam pairs between the handover vehicle and the target mmW-RRU will be tested one by one so as to find out the best one. Suppose one beam alignment test costs $10 \mu \mathrm{s}$, then given the numbers of beams at vehicles and mmW-RRUs we can get the time consumption of the conventional handover decision-making scheme. For the proposed KNN ML handover decision-making scheme, we obtain the time consumption through MATLAB programming. From Table II, it can be seen that the KNN ML based scheme can greatly reduce the handover decision-making time. Notice that Table II only shows the time consumption of the handover decision-making part, instead of the whole handover process. 


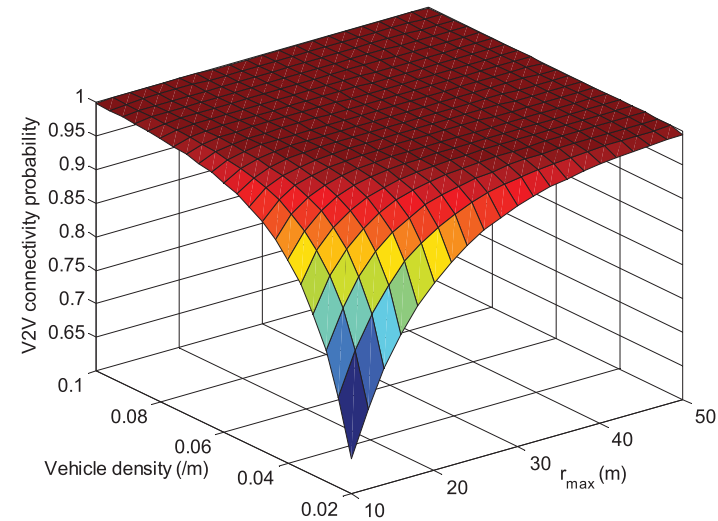

Fig. 8. Connectivity probability of $\mathrm{V} 2 \mathrm{~V}$ links.

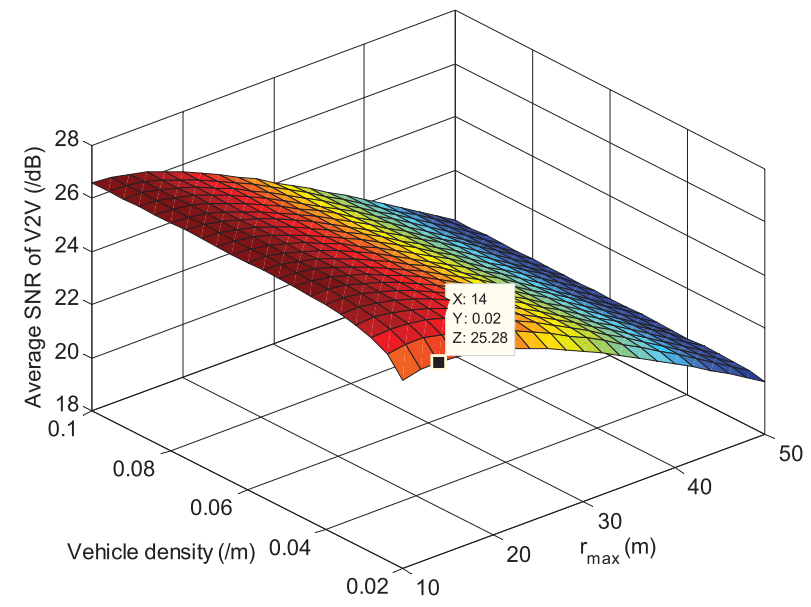

Fig. 9. Average SNR of V2V links.

\section{Performance of V2V Assisted Data Forwarding}

In this section, the performance of $\mathrm{V} 2 \mathrm{~V}$ links, including connectivity probability, average SNR and interruption probability, are presented to validate the feasibility of using V2V links to forward data during V2I handovers. In Fig. 8, the connectivity probability of $\mathrm{V} 2 \mathrm{~V}$ links versus the vehicle density and the maximum communication distance of $\mathrm{V} 2 \mathrm{~V}$ links is shown. The results match well with the intuition that the higher the vehicle density and the maximum communication distance of $\mathrm{V} 2 \mathrm{~V}$ links are, the higher the probability of finding an available $\mathrm{V} 2 \mathrm{~V}$ link for data forwarding can be achieved.

The average SNR results of V2V links during V2I handovers are given in Fig. 9. With increasing vehicle densities, the physical distance between two connected vehicles decreases equivalently. Since in this paper direct LOS V2V links are considered, shorter physical distance results in lower path loss and higher average SNR. Besides, as shown in Fig. 9, under the parameter settings in Table I, when $r_{\max }$ is shorter than $14 \mathrm{~m}$, the average SNR of V2V links rises with increasing $r_{\max }$. Nevertheless, since larger signal propagation distances can cause higher path loss, as shown in Fig. 9, when $r_{\max }$ exceeds $14 \mathrm{~m}$, the average SNR decreases with increasing $r_{\max }$.

In Fig. 10, the interruption probability of $\mathrm{V} 2 \mathrm{~V}$ links, defined as the probability that the holding time of $\mathrm{V} 2 \mathrm{~V}$ links is shorter than the duration of V2I handovers, is depicted. During the

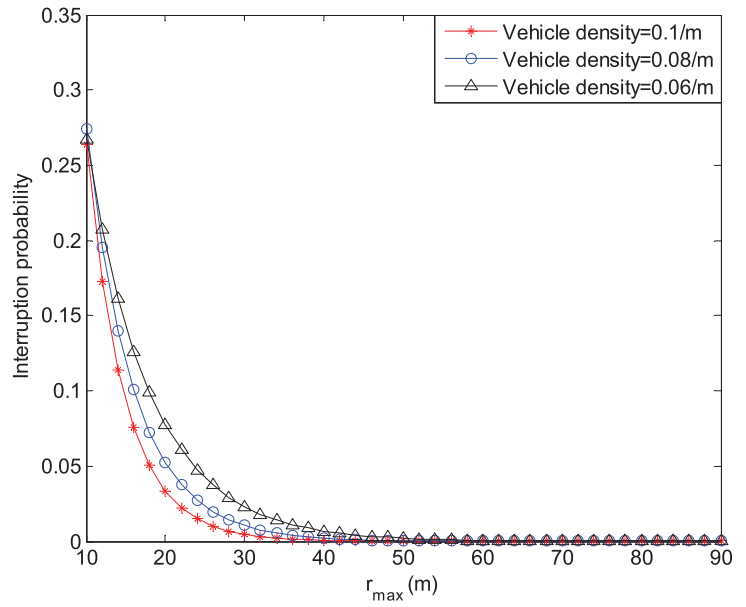

Fig. 10. Interruption probability of $\mathrm{V} 2 \mathrm{~V}$ links.

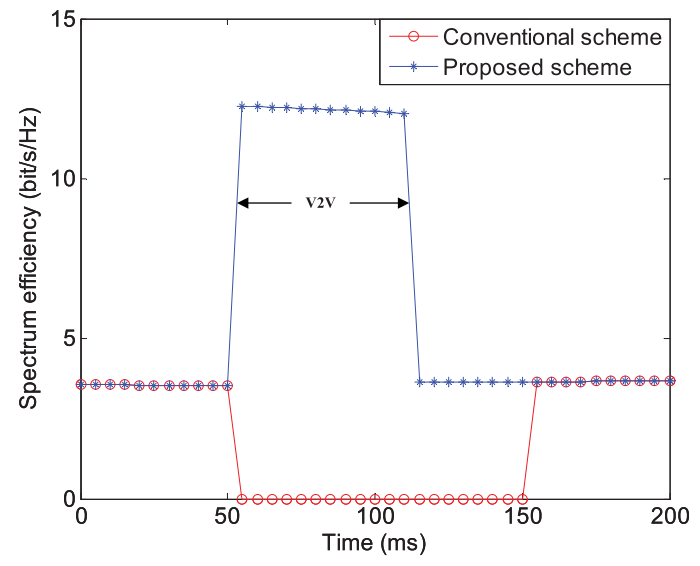

Fig. 11. Spectrum efficiency in V2I handover areas.

simulation, without loss of generality, the handover and partner vehicle are set on lane 0 and lane 1, respectively. From an overall point of view, with increasing $r_{\max }$, the interruption probability shows a downward trend, which aligns to the fact that the larger the $r_{\max }$ is, the longer the $\mathrm{V} 2 \mathrm{~V}$ link can hold. Simulation results also show that the higher the vehicle density is, the lower the interruption probability is, which aligns to the fact that with higher vehicle density, handover vehicles have more opportunities to establish V2V links for data forwarding, thereby leading to lower interruption probability. Additionally, from Fig. 10, we can observe that the higher the vehicle density is, the faster the interruption probability decreases with the rise of $r_{\max }$.

In Fig. 11, the U-plane spectrum efficiency during handovers is compared between the two different schemes. As shown in our previous work [38], in conventional hard handovers, the U-plane data transmission is cut off when the handover is triggered and will be reconnected after the U-plane path is successfully switched to a target base station. In conventional wireless communication networks without involving the directional beamforming technique, the interruption time of the U-plane data transmission during hard handovers is about $60 \mathrm{~ms}$. Even worse, in mmWave directional communication systems, we still need to take account of the time of beam training within which the U-plane is also out of connection. In this simulation, the numbers of beams at vehicles and 
mmW-RRUs are both set to 64 . To avoid transmission interruptions, in the proposed scheme existing V2V links are employed to help forward U-plane data during handovers. As shown in Fig. 11, since the relative distance between two vehicles is much smaller, V2V links can highly improve the transmission performance in the handover overlapping area.

\section{CONCLUSION}

In this paper, to enhance the robustness of mmWave vehicular networks, we have applied the C/U-plane decoupled network architecture to combine high-quality sub- $6 \mathrm{GHz}$ bands and broadband mmWave bands to guarantee the coverage performance while augmenting capacity. To address the target discovery difficulty during mmWave V2I handovers due to the blindness of narrow beams, we have proposed to utilize the CSI of sub- $6 \mathrm{GHz}$ bands and the Kernel-based ML algorithm to predict vehicles' positions and use this information to pre-activate target mmW-RRUs. To achieve fast handovers, we have developed a KNN based handover decision-making scheme to accelerate handovers. Additionally, in order to realize soft handovers, $\mathrm{V} 2 \mathrm{~V}$ links are engaged to forward service data for V2I links during handovers. Theoretical and simulation results have demonstrated the feasibility of these proposed schemes.

\section{REFERENCES}

[1] Y. Li, Q. Luo, J. Liu, H. Guo, and N. Kato, "TSP security in intelligent and connected vehicles: Challenges and solutions," IEEE Wireless Commun., vol. 26, no. 3, pp. 125-131, Jun. 2019. doi: 10.1109/MWC.2019.1800289.

[2] C.-X. Wang et al., "Cellular architecture and key technologies for 5G wireless communication networks," IEEE Commun. Mag., vol. 52, no. 2 , pp. 122-130, Feb. 2014.

[3] J. Wang, J. Liu, and N. Kato, "Networking and communications in autonomous driving: A survey," IEEE Commun. Surveys Tuts., vol. 21, no. 2, pp. 1243-1274, 2nd Quart., 2019.

[4] P. Zhou, X. Fang, Y. Fang, Y. Long, R. He, and X. Han, "Enhanced random access and beam training for millimeter wave wireless local networks with high user density," IEEE Trans. Wireless Commun., vol. 16, no. 12, pp. 7760-7773, Dec. 2017.

[5] L. Yan, X. Fang, and Y. Fang, "Control and data signaling decoupled architecture for railway wireless networks," IEEE Wireless Commun., vol. 22, no. 1, pp. 103-111, Feb. 2015.

[6] P. Wang, B. Di, H. Zhang, K. Bian, and L. Song, "Cellular V2X communications in unlicensed spectrum: Harmonious coexistence with VANET in 5G systems," IEEE Trans. Wireless Commun., vol. 17, no. 8, pp. 5212-5224, Aug. 2018.

[7] Y. S. Satriawan, C. Machbub, and E. M. I. Hidayat, "Comparison of prediction methods for moving objects in 3D coordinates using Kalman filter and least square," in Proc. 6th Int. Conf. Syst. Eng. Technol. (ICSET), Bandung, Indonesia, Oct. 2016, pp. 128-131.

[8] C. G. Prevost, A. Desbiens, and E. Gagnon, "Extended Kalman filter for state estimation and trajectory prediction of a moving object detected by an unmanned aerial vehicle," in Proc. Amer. Control Conf., New York, NY, USA, Jul. 2007, pp. 1805-1810.

[9] L. Liang, H. Peng, G. Y. Li, and X. Shen, "Vehicular communications: A physical layer perspective," IEEE Trans. Veh. Technol., vol. 66, no. 12, pp. 10647-10659, Dec. 2017.

[10] M. Roshanaei and M. Maleki, "Dynamic-KNN: A novel locating method in WLAN based on angle of arrival," in Proc. IEEE Symp. Ind. Electron. Appl., Kuala Lumpur, Malaysia, vol. 2, Oct. 2009, pp. 722-726.

[11] Y. Li, B. Cao, and C. Wang, "Handover schemes in heterogeneous LTE networks: Challenges and opportunities," IEEE Wireless Commun., vol. 23, no. 2, pp. 112-117, Apr. 2016.

[12] K. Zheng, Q. Zheng, P. Chatzimisios, W. Xiang, and Y. Zhou, "Heterogeneous vehicular networking: A survey on architecture, challenges, and solutions," IEEE Commun. Surveys Tuts., vol. 17, no. 4, pp. 2377-2396, 4th Quart., 2015.
[13] H. Ding, X. Li, Y. Cai, B. Lorenzo, and Y. Fang, "Intelligent data transportation in smart cities: A spectrum-aware approach," IEEE/ACM Trans. Netw., vol. 26, no. 6, pp. 2598-2611, Dec. 2018.

[14] Y. Wu, X. Fang, and X. Wang, "Mobility management through scalable C/U-plane decoupling in IoV networks," IEEE Commun. Mag., vol. 57, no. 2, pp. 122-129, Feb. 2019.

[15] S. Mahfouz, F. Mourad-Chehade, P. Honeine, J. Farah, and H. Snoussi, "Kernel-based machine learning using radio-fingerprints for localization in WSNs," IEEE Trans. Aerosp. Electron. Syst., vol. 51, no. 2, pp. 1324-1336, Apr. 2015.

[16] Z. Yang, Z. Zhou, and Y. Liu, "From RSSI to CSI: Indoor localization via channel response," ACM Comput. Surv., vol. 46, no. 2, Nov. 2018, Art. no. 25 .

[17] X. Wang, L. Gao, S. Mao, and S. Pandey, "CSI-based fingerprinting for indoor localization: A deep learning approach," IEEE Trans. Veh Technol., vol. 66, no. 1, pp. 763-776, Jan. 2017.

[18] A. Noerpel and Y.-B. Lin, "Handover arrangement for a PCS network," IEEE Pers. Commun., vol. 4, no. 6, pp. 18-24, Dec. 1997.

[19] Y. Lee, B. Shin, J. Lim, and D. Hong, "Effects of time-to-trigger parameter on handover performance in SON-based LTE systems," in Proc. 16th Asia-Pacific Conf. Commun. (APCC), Auckland, New Zealand, Oct./Nov. 2010, pp. 492-496.

[20] W. Luo, X. Fang, M. Cheng, and X. Zhou, "An optimized handover trigger scheme in LTE systems for high-speed railway," in Proc. 5th Int. Workshop Signal Design Appl. Commun., Guilin, China, Oct. 2011, pp. 193-196.

[21] D. Castro-Hernandez and R. Paranjape, "Optimization of handover parameters for LTE/LTE-A in-building systems," IEEE Trans. Veh. Technol., vol. 67, no. 6, pp. 5260-5273, Jun. 2018.

[22] F. B. Mismar and B. L. Evans, "Partially blind handovers for mmWave new radio aided by sub-6 GHz LTE signaling," in Proc. IEEE Int. Conf. Commun. Workshops (ICC Workshops), May 2018, pp. 1-5.

[23] S.-M. Oh, S.-Y. Kang, K.-C. Go, J.-H. Kim, and A.-S. Park, "An enhanced handover scheme to provide the robust and efficient interbeam mobility," IEEE Commun. Lett., vol. 19, no. 5, pp. 739-742, May 2015.

[24] J. Zhu, X. Luo, and D. Chen, "Maximum likelihood scheme for fingerprinting positioning in LTE system," in Proc. IEEE 14th Int. Conf. Commun. Technol., Chengdu, China, Nov. 2012, pp. 428-432.

[25] A. Paier, D. Faetani, and C. F. Mecklenbräuker, "Performance evaluation of IEEE $802.11 \mathrm{p}$ physical layer infrastructure-to-vehicle real-world measurements," in Proc. 3rd Int. Symp. Appl. Sci. Biomed. Commun. Technol. (ISABEL), Roma, Italy, Nov. 2010, pp. 1-5.

[26] S. Mahfouz, F. Mourad-Chehade, P. Honeine, J. Farah, and H. Snoussi, "Target tracking using machine learning and Kalman filter in wireless sensor networks," IEEE Sensors J., vol. 14, no. 10, pp. 3715-3725, Oct. 2014.

[27] B. Schölkopf, R. Herbrich, and A. J. Smola, "A generalized representer theorem," in Proc. Int. Conf. Comput. Learn. Theory, Berlin, Germany, Jul. 2001, pp. 416-426.

[28] W. Li, P. Yi, Y. Wu, L. Pan, and J. Li, "A new intrusion detection system based on KNN classification algorithm in wireless sensor network," J. Elect. Comput. Eng., vol. 2014, no. 5, pp. 1-8, 2014.

[29] D. Moltchanov, "Distance distributions in random networks," Ad Hoc Netw., vol. 10, no. 6, pp. 1146-1166, Aug. 2012.

[30] J. Liu, H. Nishiyama, N. Kato, and J. Guo, "On the outage probability of device-to-device-communication-enabled multichannel cellular networks: An RSS-threshold-based perspective," IEEE J. Sel. Areas Commun., vol. 34, no. 1, pp. 163-175, Jan. 2016.

[31] P. J. Clark and F. C. Evans, "Distance to nearest neighbor as a measure of spatial relationships in populations," Ecology, vol. 35, no. 4 pp. 445-453, Oct. 1954.

[32] C. F. Mecklenbrauker et al., "Vehicular channel characterization and its implications for wireless system design and performance," Proc. IEEE, vol. 99, no. 7, pp. 1189-1212, Jul. 2011.

[33] T. S. Rappaport, Wireless Communications: Principles and Practice. Upper Saddle River, NJ, USA: Prentice-Hall, 1999.

[34] V. Erceg, P. Soma, D. S. Baum, and S. Catreux, "Multiple-input multipleoutput fixed wireless radio channel measurements and modeling using dual-polarized antennas at $2.5 \mathrm{GHz}$," IEEE Trans. Wireless Commun., vol. 3, no. 6, pp. 2288-2298, Nov. 2004

[35] J. Kim, H.-S. Chung, I. G. Kim, H. Lee, and M. S. Lee, "A study on millimeter-wave beamforming for high-speed train communication," in Proc. Int. Conf. Inf. Commun. Technol. Converg. (ICTC), Jeju, South Korea, Oct. 2015, pp. 1190-1193. 
[36] A. Tassi, M. Egan, R. J. Piechocki, and A. Nix, "Modeling and design of millimeter-wave networks for highway vehicular communication," IEEE Trans. Veh. Technol., vol. 66, no. 12, pp. 10676-10691, Dec. 2017.

[37] R. Ismayilov, M. Kaneko, T. Hiraguri, and K. Nishimori, "Adaptive beam-frequency allocation algorithm with position uncertainty for millimeter-wave MIMO systems," in Proc. IEEE 87th Veh. Technol. Conf. (VTC Spring), Porto, Portugal, Jun. 2018, pp. 1-5.

[38] L. Yan, X. Fang, and Y. Fang, "A novel network architecture for $\mathrm{C} / \mathrm{U}$-plane staggered handover in $5 \mathrm{G}$ decoupled heterogeneous railway wireless systems," IEEE Trans. Intell. Transp. Syst., vol. 18, no. 12, pp. 3350-3362, Dec. 2017.

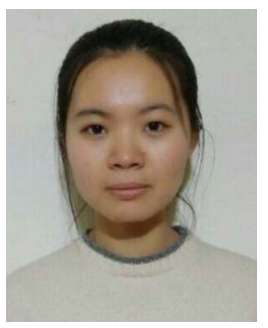

Li Yan received the B.E. degree in communication engineering and the Ph.D. degree in communication and information systems from Southwest Jiaotong University, China, in 2012 and 2018, respectively. She was a Visiting Student at the Department of Electrical and Computer Engineering, University of Florida, Gainesville, FL, USA, from 2017 to 2018. She is currently a Lecturer with Southwest Jiaotong University.

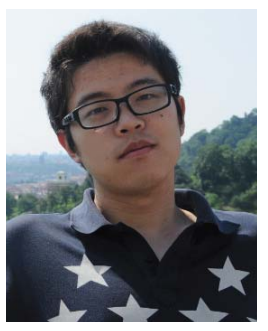

Haichuan Ding received the B.Eng. and M.S degrees in electrical engineering from the Beijing Institute of Technology (BIT), Beijing, China, in 2011 and 2014, respectively, and the Ph.D. degree in electrical and computer engineering from the University of Florida, Gainesville, FL, USA, in 2018. From 2012 to 2014, he was with the Department of Electrical and Computer Engineering, University of Macau, as a Visiting Student. Since 2019, he has been a Post-Doctoral Research Fellow with the Department of Electrical Engineering and Computer Science, University of Michigan, Ann Arbor, MI, USA. His current research interests include mmWave and V2X communications.

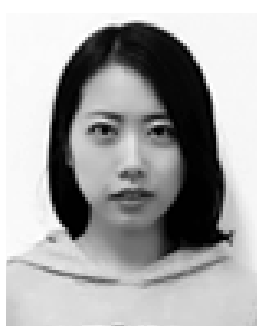

Lan Zhang received the B.S. and M.S. degrees from the University of Electronic Science and Technology of China (UESTC), in 2013 and 2016, respectively. She is currently pursuing the Ph.D. degree with the Department of Electrical and Computer Engineering, University of Florida.

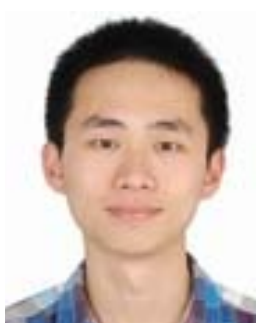

Jianqing Liu received the B.E. degree from the University of Electronic Science and Technology of China in 2013, and the Ph.D. degree from the University of Florida in 2018. He is currently a tenure-track Assistant Professor with the Department of Electrical and Computer Engineering, The University of Alabama in Huntsville. He was a recipient of the 2018 Best Journal Paper Award from the IEEE Technical Committee on Green Communications and Computing (TCGCC) and the Best Paper Award from the 2012 IEEE Workshop on MMWCST.

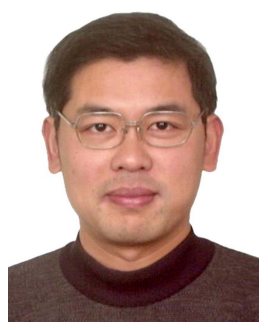

Xuming Fang (SM'16) received the B.E. degree in electrical engineering, the M.E. degree in computer engineering, and the $\mathrm{Ph} . \mathrm{D}$. degree in communication engineering from Southwest Jiaotong University, Chengdu, China, in 1984, 1989, and 1999, respectively. He was a Faculty Member with the Department of Electrical Engineering, Tongji University, Shanghai, China, in September 1984. He then joined the School of Information Science and Technology, Southwest Jiaotong University, where he has been a Professor since 2001, and the Chair of the Department of Communication Engineering since 2006. He held visiting positions with the Institute of Railway Technology, Technical University at Berlin, Berlin, Germany, in 1998 and 1999, respectively, and with the Center for Advanced Telecommunication Systems and Services, University of Texas at Dallas, Richardson, in 2000 and 2001, respectively. He has, to his credit, around 200 high-quality research papers in journals and conference publications. He has authored or coauthored five books or textbooks. He is the Chair of the IEEE Vehicular Technology Society of Chengdu Chapter.

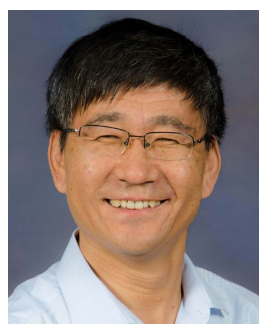

Yuguang Fang (F'08) received the M.S. degree from Qufu Normal University, Shandong, China, in 1987, the Ph.D. degree from Case Western Reserve University in 1994, and the Ph.D. degree from Boston University in 1997. He joined the Department of Electrical and Computer Engineering, University of Florida as an assistant professor in 2000, was promoted to an associate professor in 2003 and a full professor in 2005, and has been a distinguished professor, since 2019.

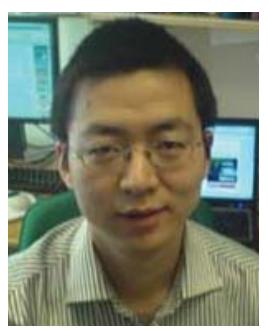

Ming Xiao (S'02-M'07-SM'12) received the bachelor's and master's degrees in engineering from the University of Electronic Science and Technology of China, Chengdu, in 1997 and 2002, respectively, and the Ph.D. degree from the Chalmers University of Technology, Sweden, in November 2007. From 1997 to 1999 , he worked as a Network and Software Engineer at ChinaTelecom. Since November 2007, he has been with the Department of Information Science and Engineering, School of Electrical Engineering and Computer Science, Royal Institute of Technology, Sweden, where he is currently an Associate Professor. Since 2012, he has been an Associate Editor of the IEEE TRANSACTIONS ON COMmunications, the IEEE COMmunications LetTers (Senior Editor, since 2015), and the IEEE WIRELESS COMMUNICATIONS LETTERS (2012-2016). He was the lead Guest Editor for the IEEE JSAC Special Issue on "Millimeter Wave Communications for Future Mobile Networks" in 2017.

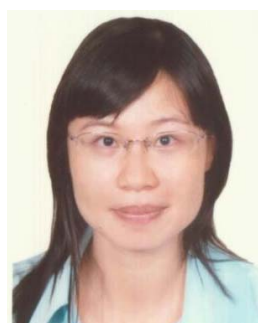

Xiaoxia Huang (M'07) received the B.E. and M.E. degrees in electrical engineering from the Huazhong University of Science and Technology, Wuhan, China, in 2000 and 2002, respectively, and the Ph.D. degree in electrical and computer engineering from the University of Florida, Gainesville, FL, USA, in 2007. She is currently a Professor with the School of Electronics and Communication Engineering, Sun Yat-Sen University. 